\title{
An Adaptive Indexing Technique Using Spatio-Temporal Query Workloads
}

\author{
Hyung-Ju Cho, Jun-Ki Min, Chin-Wan Chung \\ Dept. of Electrical Engineering 83 Computer Science \\ Korea Advanced Institute of Science and Technology \\ 373-1 Kusong-dong, Yusong-gu, Taejon 305-701, Korea \\ \{hjcho, jkmin, chungcw\}@islab.kaist.ac.kr
}

\begin{abstract}
Many spatio-temporal access methods, such as the HR-tree, the 3DR-tree, and the MV3R-tree, have been proposed for timestamp and interval queries. However, these access methods have the following problems: the poor performance of the 3DR-tree for timestamp queries, the huge size and the poor performance of the HR-tree for interval queries, and the large size and the high update cost of the MV3R-tree. We address these problems by proposing an adaptive partitioning technique called the Adaptive Partitioned R-tree (APR-tree) using workloads with timestamp and interval queries. The APR-tree adaptively partitions the time domain using query workloads. Since the time domain of the APR-tree is automatically fitted to query workloads, the APR-tree outperforms the other access methods for various query workloads. The size of the APR-tree is on the average 1.3 times larger than that of the 3DR-tree which has the smallest size. The update cost of the APR-tree is on the average similar to that of the 3DR-tree which has the smallest update cost.
\end{abstract}

Key words: Spatio-Temporal Databases, Indexing Technique, R-trees, Timestamp and Interval Queries

\section{Introdcution}

Spatio-temporal databases manipulate moving objects whose positions and shapes may change along with time. In other words, they are said to be the combination of spatial databases and temporal databases. To support trajectory (or navigational) queries, timestamp (or timeslice) queries, and interval queries, many spatio-temporal access methods have been proposed. They can be classified into two groups. One consists of access methods for future queries, 


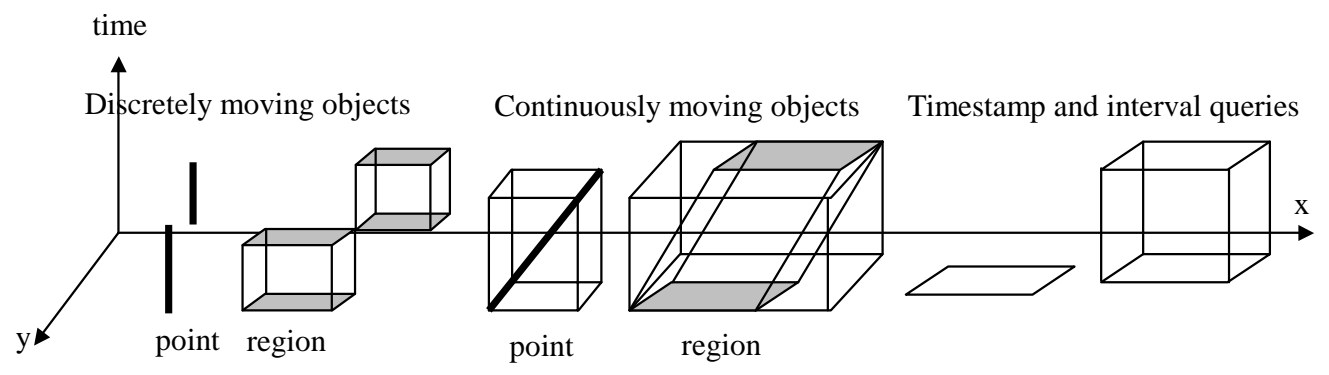

Fig. 1. Moving objects and timestamp/interval queries

and the other for historical queries. Although timestamp and interval queries are available to retrieve the future information of moving objects, in this paper, we focus on timestamp and interval queries for the past information. Note that time is represented as discrete timestamps $[3,8,9,11]$. Timestamp queries retrieve all the objects intersecting a spatial window at a specific timestamp, and interval queries include multiple consecutive timestamps. Therefore, the timestamp query can be considered as a special case of the interval query, where the interval contains only one timestamp. Access methods for future queries focus on predicting the future locations of objects by storing their current positions, velocities, and directions [5-7]. Trajectory queries mainly deal with the navigational trajectories of particular objects. The STR-tree [8] and the TB-tree [8] belong to the access methods for trajectory queries.

Moving objects can be classified according to the spatial shapes and the temporal movements of objects $[1,9,11,12]$. Their spatial shapes are approximated by minimum bounding rectangles (MBRs). Their temporal movements are either discrete or continuous. However, the problem of indexing moving objects can be reduced to indexing discrete rectangles that contain both their spatial shapes and time intervals. Figure 1 illustrates this. The R-tree can be a good candidate for indexing these rectangles. Timestamp and interval queries can also be represented as rectangles. Therefore, timestamp and interval queries can be considered as range queries of the R-tree. However, the time region monotonically increases along with time while the spatial region does not. Therefore, if a single R-tree covers the entire time region, the performance of the R-tree would degrade for timestamp and interval queries since the time region enlarges as time passes.

In this paper, we concentrate on timestamp and interval queries for moving objects with historical information such as vehicles with GPSs, people with mobile devices, planes and ships on a voyage. Many spatio-temporal index structures have been developed for timestamp and interval queries during the past few years $[3,10,11]$. If a single index structure covers the entire time region, its performance would degrade since the indexed time region enlarges as time passes. Therefore, some spatio-temporal access methods partitions the whole time region into multiple time regions indexed by the corresponding $\mathrm{R}$ - 
trees. However, since no existing spatio-temporal access methods utilize query workoads, they can neither support a variety of query workloads efficiently nor reflect the change of the query workload along with time. We address these problems by proposing the Adaptive Partitioned R-tree (APR-tree) which automatically adapts to workloads with timestamp and interval queries. Based on query workloads, the APR-tree adaptively partitions the time region. Our contributions are summarized as follows:

- The APR-tree achieves the best performance for various workloads with timestamp and interval queries except for workloads with $100 \%$ timestamp queries. Its performance is on the average $40 \%$ better than that of the MV3R-tree.

- Although the size of the APR-tree is affected by query workloads, the size of the APR-tree is on the average 1.3 times larger than that of the 3DRtree which has the smallest size and does not exceed 2 times that of the 3DR-tree.

- Although the update cost of the APR-tree are also affected by query workloads, the update cost of the APR-tree is on the average similar to that of the 3DR-tree which has the smallest update cost.

- Through mathematical analyses and extensive experimental study, we show that the APR-tree deals with various query workloads more efficiently than the other access methods.

The rest of the paper is organized as follows: Section 2 surveys the existing access methods for timestamp and interval queries. Section 3 describes the APR-tree, and the corresponding insertion and query processing algorithms. Section 4 provides the optimal jurisdiction interval length of the APR-tree based on mathematical analyses of the performance and the size of the APRtree. Section 5 presents experimental results over various query workloads. Finally, Section 6 provides conclusions.

\section{Related Work}

In this section, we describe structures, and strong and weak points of existing access methods, the 3DR-tree, the HR-tree, and the MV3R-tree.

\section{$2.13 D R$-trees}

The 3DR-tree [10] simply considers time as another dimension of the Rtree $[15,16]$. Whenever an object moves to another position or changes its shape, a new MBR is created to represent the change of the object and the 


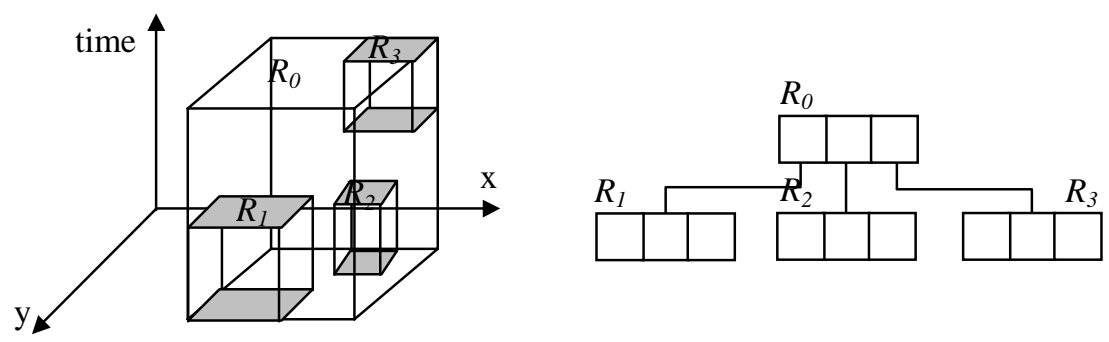

Fig. 2. An example of the 3DR-tree

MBR containing both its spatial extent and lifespan is inserted into the 3DRtree. This MBR is effective up to the next change of the object. Figure 2 shows an example of the 3DR-tree. In this figure, $R_{1}, R_{2}$, and $R_{3}$ represent MBRs for the movements of objects, and $R_{0}$ contains $R_{1}, R_{2}$ and $R_{3}$. As time passes, the time region enlarges accordingly. This makes the performance of the 3DR-tree degrade because in the 3DR-tree, an R-tree keeps the whole time region.

Also, long-lived records cause a huge dead space which is a part of the MBR, but does not cover any record. Therefore, they degrade the query performance of the 3DR-tree. However, the 3DR-tree has the smallest size since the 3DRtree has no duplicate data unlike the HR-tree and the MV3R-tree.

\subsection{HR-trees}

The Historical R-tree (HR-tree) [3] creates an R-tree whenever objects in the previous R-tree change their positions or shapes, but common branches of consecutive R-trees are stored only once in order to save space.

The timestamp query is directed to the corresponding R-tree and the search is performed inside this tree only. Thus the timestamp query becomes the ordinary window query and is handled very efficiently. The interval query should search the corresponding R-trees of all the timestamps involved. Even when only one object has changed its position, the HR-tree may update nodes contained in the path between the leaf node corresponding to the object and the root node. Therefore, the size of the HR-tree is several times larger than that of the 3DR-tree. Figure 3 shows an example of the HR-tree. $R_{1}\left[t_{1}, t_{2}\right)$ deals with records whose time intervals are contained in time interval $\left[t_{1}, t_{2}\right)$ and $R_{2}\left[t_{2}, t_{3}\right)$ manipulates records whose time intervals are contained in $\left[t_{2}, t_{3}\right)$. Suppose that object $f$ changes its position to $f^{\prime}$ from $B_{0}$ to $C_{0}$ at $t_{2}$. The three nodes $B_{0}, C_{0}, D_{0}$ which are associated with $f$ and $f^{\prime}$ are copied and updated to three new nodes $B_{1}, C_{1}, D_{1}$ in the gray color. Note that the unchanged other objects except $f$ and $f^{\prime}$ are shared between $R_{1}$ and $R_{2}$. 


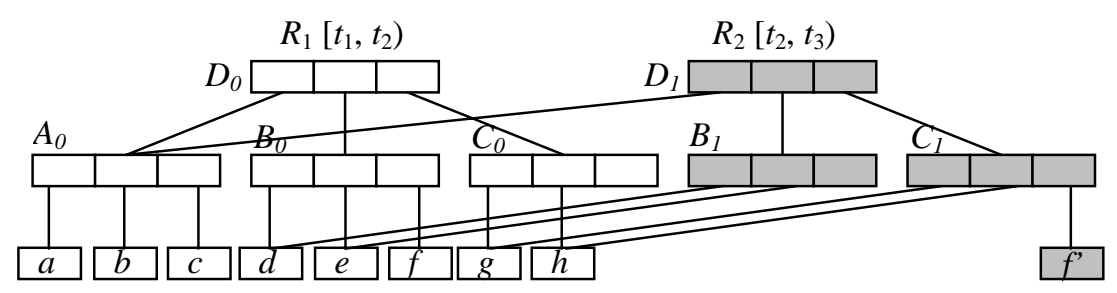

Fig. 3. An example of the HR-tree

\subsection{MV3R-trees}

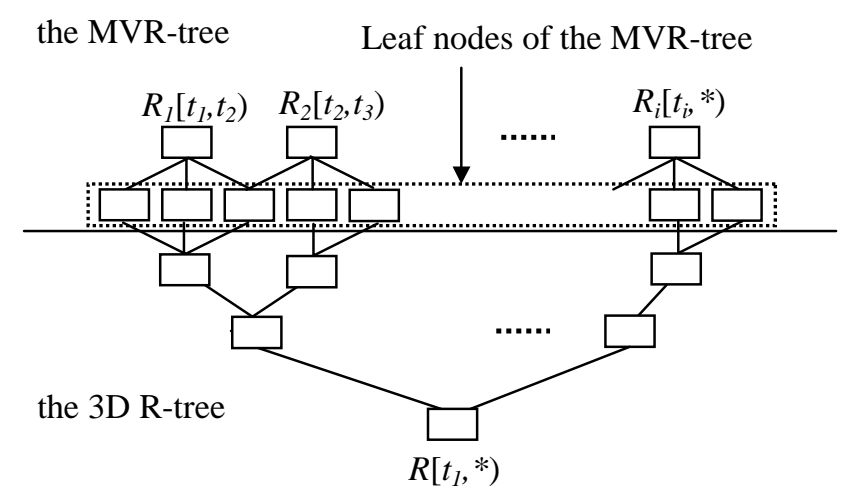

Fig. 4. The structure of the MV3R-tree

The Multi-Version 3DR-tree (MV3R-tree) [11] is the access structure that combines the Multi-Version R-tree (MVR-tree) and a small auxiliary 3DRtree built on the leaf nodes of the MVR-tree. The former is used to answer timestamp and short interval queries and the latter to answer long interval queries. Figure 4 illustrates the structure of the MV3R-tree. As shown in this figure, the MVR-tree consists of multiple R-trees that have their own jurisdiction intervals. For example, $R_{1}\left[t_{1}, t_{2}\right)$ deals with records whose time intervals belong to $\left[t_{1}, t_{2}\right)$ while $R_{2}\left[t_{2}, t_{3}\right)$ manipulates records whose time intervals belong to $\left[t_{2}, t_{3}\right)$.

Although the size of the auxiliary 3DR-tree is very small since it shares the leaf nodes of the MVR-tree, it improves the performance on interval queries and provides flexibility to algorithms for processing other spatial queries such as the join and the $k$-nearest neighbor.

However, the MV3R-tree has a few disadvantages. The size of the MV3R-tree is typically 1.5 times larger than that of the 3DR-tree due to many duplicate data from version splits [4]. In addition, the update cost of the MV3R-tree is much higher than those of the HR-tree and the 3DR-tree. They maintain one structure, while the MV3R-tree has to modify two structures simultaneously when records are inserted. The auxiliary 3DR-tree suffers from the large search space for interval queries since it is also responsible for the whole history like the 3DR-tree. 


\subsection{Comparison between the 3DR-tree and the HR-tree}

One of the most significant differences between the HR-tree and the 3DR-tree is the average jurisdiction interval length of R-trees which form each structure. In the HR-tree, R-trees are almost created every timestamp and each R-tree is responsible for the information of the specific timestamp only. In the 3DRtree, an R-tree is created only once, and this R-tree keeps all the information of the whole time range. Figure 5 illustrates this observation.

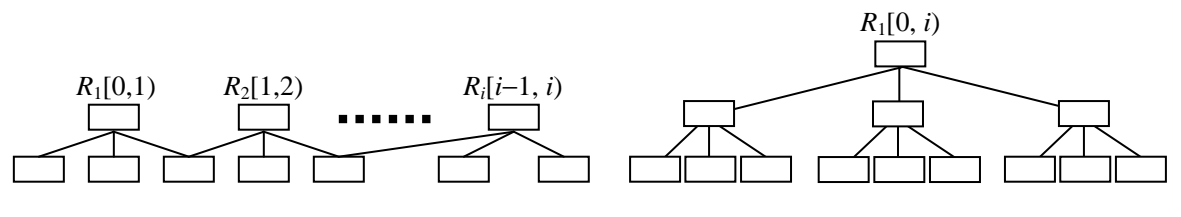

(a) an HR-tree

(b) a 3DR-tree

Fig. 5. Structures of the HR-tree and the 3DR-tree

In the HR-tree, the timestamp query is evaluated by the R-tree which corresponds to the timestamp of this query. In the 3DR-tree, however, the timestamp query is always processed by a single R-tree that covers the whole time range. Thus, the 3DR-tree has to access much more nodes than the HR-tree due to a prohibitive search space. However, to answer interval queries, the HRtree accesses more nodes than the 3DR-tree since the HR-tree has to search many R-trees whose jurisdiction intervals overlap these interval queries. The 3DR-tree should still search the largest space irrespective of the query condition. Consequently, to overcome the poor performance of the 3DR-tree on timestamp and interval queries, it is necessary to reduce the search space, while to overcome the poor performance of the HR-tree on interval queries, it is necessary to decrease the number of R-trees which are searched.

\section{APR-Trees}

Section 3.1 presents an adaptive partitioning method based on query workloads. Next, we describe insert and search algorithms of the APR-tree in Section 3.2 .

\subsection{Query Adaptive Partitioning Method}

To overcome the problem with the 3DR-tree, we apply the query-adaptive partitioning method to the 3DR-tree. We call the resultant structure the Adaptive Partitioned R-tree (APR-tree). The APR-tree consists of multiple 3DR-trees, 
each of which is responsible for the fixed time interval derived from query workloads. Since several new terms and symbols are used in this work, they are summarized in Table 1.

Table 1

Summary of symbols and terms

\begin{tabular}{|c|l|}
\hline Symbol or Term & \multicolumn{1}{|c|}{ Description } \\
\hline \hline$*$ & the special reserved word "now". \\
\hline$A V G_{\text {records }}$ & the average lifespan length of records. \\
\hline$A V G_{\text {queries }}$ & the average time interval length of queries. \\
\hline$R_{i}\left[t_{i}, t_{i+1}\right)$ & the R-tree that is created the $i$-th and covers the time interval of $\left[t_{i}, t_{i+1}\right)$. \\
\hline$L_{i}$ & the jurisdiction interval length of $R_{i}\left[t_{i}, t_{i+1}\right)$. Namely, $L_{i}=t_{i+1}-t_{i}$. \\
\hline$K$ & the number of $R_{i}$ 's. \\
\hline$C_{i}$ & the cardinality of objects with distinct identifiers which belong to $R_{i}$. \\
\hline$F$ & the average fanout of a node. \\
\hline$H_{i}$ & the height of $R_{i}$. \\
\hline$N_{i}$ & the number of records in $R_{i}$. \\
\hline$N$ & the total number of records. \\
\hline$M_{h}$ & the number of nodes at height $h$. \\
\hline$a_{h}$ & the average area covered by a node at height $h$. \\
\hline$D$ & the number of dimensions. \\
\hline Size $(R-$ tree $)$ & the size of the R-tree. \\
\hline$A J I L(R-$ tree $)$ & the average length of the time ranges covered by R-trees in the structure. \\
\hline
\end{tabular}

The average jurisdiction interval length (AJIL) is introduced to explain the property of the APR-tree. AJIL $(R-$ tree $)$ can be defined as follows:

$$
A J I L(R-\text { tree })=\frac{1}{K} \cdot \sum_{i=1}^{K} L_{i}
$$

where $K$ is the number of $R_{i}$ 's and $L_{i}$ is the jurisdiction interval length of $R_{i}$. In the case of the MVR-tree in Figure 6, $A J I L(M V R-$ tree $)=\frac{10+11+10+9}{4}=10$ since $K$ is 4 , and $L_{1}, L_{2}, L_{3}$, and $L_{4}$ are 10 (=10-0), 11 (=21-10), 10 $(=31-21)$, and $9(=40-31)$, respectively. Since $K=1$ in the 3DR-tree, $A J I L(3 D R-$ tree $)=40(=40-0)$. In the HR-tree, AJIL $(H R-$ tree $) \cong 1$ since R-trees may be created every timestamp.

Table 2 summarizes $A J I L(R-$ tree $)$ of each structure. The search space of queries is affected by $A J I L(R-$ tree $)$ of each structure. In the case of timestamp queries, $A J I L(R-$ tree $)=1$ is optimal since the timestamp query is associated with the specific timestamp. In this reason, the HR-tree achieves the best performance for timestamp queries. Similarly, in the case of interval queries, $A J I L(R-$ tree $)$ is closely associated with the interval query cost. The relationship between the interval query cost and $A J I L(R-$ tree $)$ is explained in Section 4.3. 


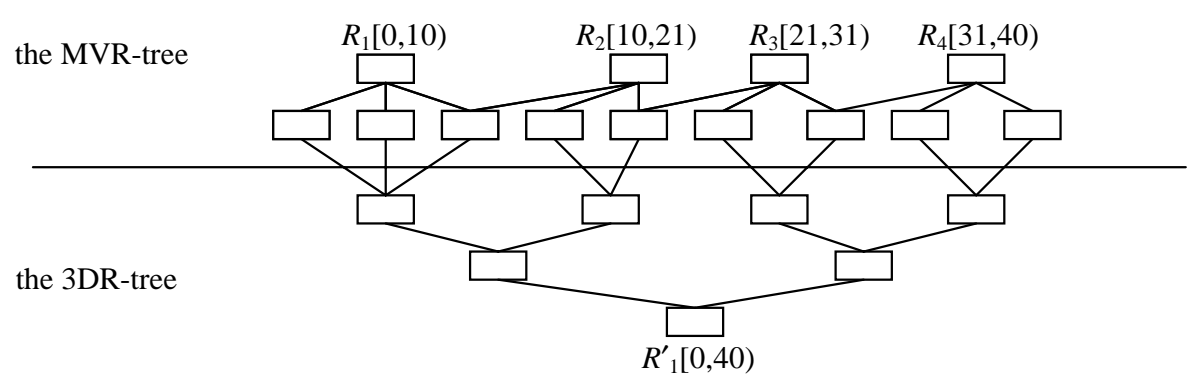

Fig. 6. $A J I L(M V R-$ tree $)=10$ and $A J I L(3 D R-$ tree $)=40$

Table 2

$A J I L(R-$ tree $)$ of each access method

\begin{tabular}{|c|l|}
\hline Access Method & \multicolumn{1}{|c|}{$\operatorname{AJIL}(R-$ tree $)$} \\
\hline \hline HR-tree & 1 \\
\hline 3DR-tree & $L_{1}$ \\
\hline MV3R-tree & $\begin{array}{l}\text { MVR-tree: } \frac{1}{K} \cdot \sum_{i=1}^{K} L_{i} \\
\text { Aux. 3DR-tree: } L_{1}\end{array}$ \\
\hline APR-tree & $\frac{1}{K} \cdot \sum_{i=1}^{K} L_{i}$ \\
\hline
\end{tabular}

Although $A J I L(A P R$-tree $)$ is similar to AJIL(MVR-tree), the APR-tree and the MVR-tree employ the very different policies of determining $L_{i}$. While $L_{i}$ of the MVR-tree is determined by version splits of root nodes, $L_{i}$ of the APR-tree is determined by query workloads. In other words, $A J I L(M V R-$ tree) depends on datasets, but $A J I L(A P R-$ tree $)$ depends on query workloads. Figure 7 depicts the structure of the APR-tree. Unlike the HR-tree and the MVR-tree, adjacent R-trees of the APR-tree do not share nodes. In this figure, let $A=<i d, s,\left[t_{\text {start }}, t_{\text {end }}\right)>$ be a record for an object's movement, where $i d$ is an object identifier, $s$ is the spatial MBR, and $t_{\text {start }}$ and $t_{\text {end }}$ are the time when the record was inserted and logically deleted, respectively. The logical deletion is to change the end time * (i.e. "now") of the lifespan of a live record to a specific time, where a live record denotes the record whose lifespan's end time is $*$. Since $A=<i d, s,\left[t_{\text {start }}, t_{\text {end }}\right)>$ intersects the boundary between $R_{1}$ and $R_{2}$, it is divided into $A_{1}=<i d, s,\left[t_{\text {start }}, t_{2}\right)>$ and $A_{2}=<i d, s,\left[t_{2}, t_{\text {end }}\right)>$, and $A_{1}$ and $A_{2}$ are inserted into $R_{1}$ and $R_{2}$, respectively. Therefore, a duplicate record occurs when the lifespan of a record intersects the boundary.

In Figure 7, $L_{1}, L_{2}$, and $L_{i}$ denote the jurisdiction interval lengths of $R_{1}, R_{2}$, and $R_{i}$, respectively, and the jurisdiction interval length of an R-tree may not be the same as those of other R-trees. This is due to the fact that the query workload may change along with time. 


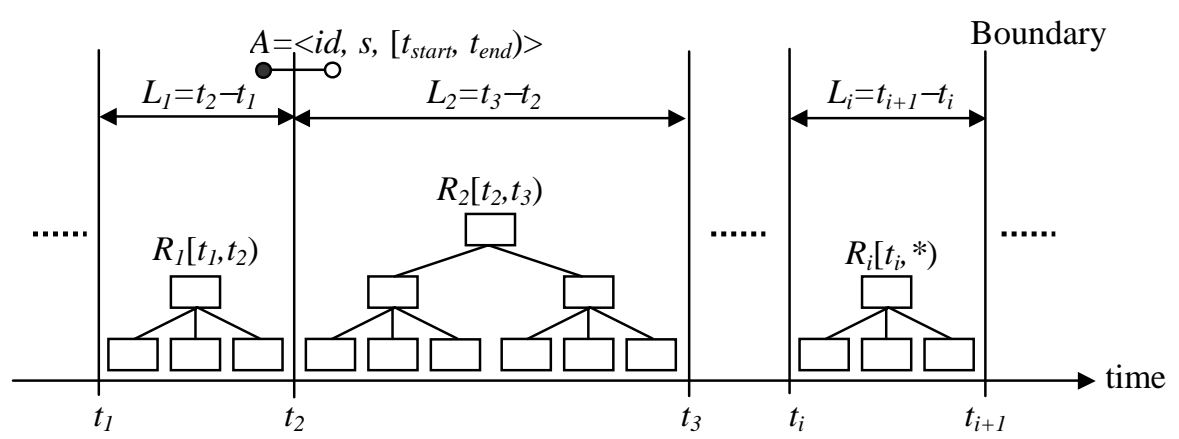

Fig. 7. The structure of the APR-tree

proc insert (record $r_{2}=<i d, s_{2},\left[t_{2}, *\right)>$ )

\section{begin}

1. $/ *$ find $R_{i}$ which contains $r_{2}$ and insert $r_{2}$ into $R_{i} * /$

2. for $R_{i}\left[t_{i}, t_{i+1}\right)$ where $i$ is from 1 to $K$ do

3. if $\left(\left[t_{i}, t_{i+1}\right)\right.$ contains $\left.\left[t_{2}, *\right)\right)$ then

4. $\quad$ insert $r_{2}$ to $R_{i}$

5. $\quad$ break $/ *$ exit for loop */

6. endif

7.

8. $/ *$ conduct the logical deletion of $r_{1}$ that is the previous record of $r_{2} * /$

9. let $r_{1}=<i d, s_{1},\left[t_{1}, *\right)>$ be the previous record of $r_{2}$

10. ${ }^{*}$ of $r_{1}$ is changed into $t_{2}$. i.e., $r_{1}=<i d, s_{1},\left[t_{1}, t_{2}\right)>$.

11.

12. /* find $R_{i}$ 's which intersect $r_{1}$ and insert $r_{1}$ into $R_{i}$ 's */

13. for $R_{i}\left[t_{i}, t_{i+1}\right)$ where $i$ is from 1 to $K$ do

14. if $\left(\left[t_{i}, t_{i+1}\right)\right.$ intersects $\left.\left[t_{1}, t_{2}\right)\right)$ then

15. insert $r_{1}$ to $R_{i}$

16. endif

end

Fig. 8. Insertion algorithm of the APR-tree

\subsection{Insertion and Search Algorithms}

If the lifespan of a record does not intersect the boundary, the record is inserted into the R-tree covering the lifespan of the record. Otherwise, after the record is divided by the boundary (this is called the data fragmentation), each of records spawned by the data fragmentation is inserted into the $\mathrm{R}$ tree whose jurisdiction interval contains the lifespan of the record. Figure 8 presents the insertion algorithm of the APR-tree. Note that the insertion of a record accompanies the logical deletion of the previous record.

Figure 9 illustrates an example of logical deletion. Record $A_{1}$ is logically 
deleted at timestamp $t_{2}$, where $s_{1}$ and $s_{2}$ indicate the spatial MBRs of $A_{1}$ and $A_{2}$, respectively, and $\left[t_{1}, t_{2}\right)$ and $\left[t_{2}, *\right)$ indicate the lifespans of $A_{1}$ and $A_{2}$, respectively.
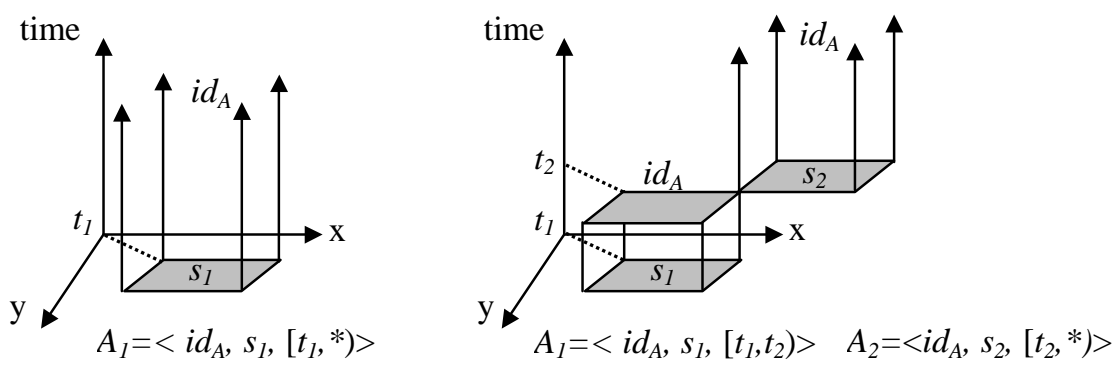

Fig. 9. Logical deletion at timestamp $t_{2}$

The timestamp and interval query processing with the APR-tree is similar to that with the 3DR-tree. However, since the 3DR-tree consists of a single tree structure, it should search the largest space. Particularly, this prohibitively degrades the performance of the 3DR-tree for timestamp queries. The APRtree outperforms the 3DR-tree for timestamp and interval queries due to the search space restriction and the reduced dead space by the data fragmentation. Like the HR-tree and the MVR-tree, the APR-tree may search several R-trees whose jurisdiction intervals overlap the time interval of the query. The search algorithm of R-trees which constitute the APR-tree is the same as that of the original R-tree $[15,16]$. As shown in Figure 10, query $Q_{1}$ is processed by $R_{i}$ and query $Q_{2}$ is processed by $R_{i}$ and $R_{i+1}$.

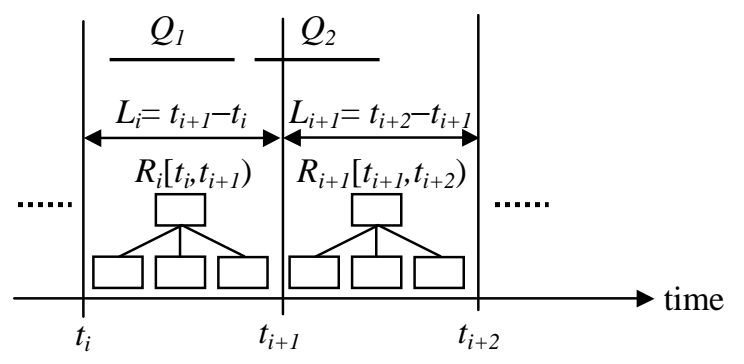

Fig. 10. Interval query processing in the APR-tree

\section{Analysis}

In this section, we assume that all the data are uniformly distributed on the $d$-dimensional work space $W S=[0,1)^{d}$ which contains spatial and temporal dimensions ("uniformity assumption" [2,14,17]). 
When the whole time domain is partitioned into the $\mathrm{K}$ time domains, the size of the APR-tree becomes the sum of the sizes of R-trees that constitute the APR-tree. Let Size $(R-$ tree $)$ denote the size of the R-tree. The size of the APR-tree is:

$$
\operatorname{Size}(A P R-\operatorname{tree})=\sum_{i=1}^{K} \operatorname{Size}\left(R_{i}\right)
$$

To compute $\operatorname{Size}\left(R_{i}\right)$, we devise the formula $\operatorname{Size}\left(R_{i}\right)$ as follows:

$$
\operatorname{Size}\left(R_{i}\right)=\sum_{h=1}^{H_{i}} \frac{T_{i} \cdot C_{i}}{f^{h}}
$$

where $T_{i}$ is the average number of records which keep the history of a moving object in $R_{i}, C_{i}$ is the cardinality of moving objects in $R_{i}, \mathrm{f}$ denotes the average fanout of a node, and $H_{i}$ denotes the height of $R_{i}$. Thus, $T_{i} \cdot C_{i}$ becomes the total number of records in $R_{i}$.

$T_{i}$ can be computed by $L_{i}$ and $A V G_{\text {records }}$, where $L_{i}$ is the jurisdiction interval length of $R_{i}$ and $A V G_{\text {records }}$ is the average lifespan length of records.

$$
T_{i}=\frac{L_{i}}{A V G_{\text {records }}}+\left(1-\frac{1}{A V G_{\text {records }}}\right)
$$

In Formula (3), $\frac{L_{i}}{A V G_{\text {records }}}$ is the average number of non-duplicate records of a moving object in $R_{i}$ and $\left(1-\frac{1}{A V G_{\text {records }}}\right)$ is the average number of duplicate records of the moving object in $R_{i}$. Whenever an object changes its position or shape, a new record is inserted into the R-tree. Therefore, the average number of records spawned by the object is $\frac{L_{i}}{A V G_{\text {records }}}$. In Figure 11(a), let $a_{1}$, $a_{2}, a_{3}$ and $a_{4}$ be the four records spawned by an object. $a_{1}$ and $a_{4}$ should be divided due to the boundary, so two duplicate records occur. However, since the boundary is shared between adjacent R-trees, one of two duplicate records belongs to $R_{i}$ and the other belongs to $R_{i-1}$ or $R_{i+1}$. Additionally, as shown in Figure 11(b), since the timestamp is discrete, there are finite cases that a record crosses the boundary. When the start time of a record's lifespan meets the boundary such as record d1, the record is not fragmented. Therefore, an object produces $\left(1-\frac{1}{A V G_{\text {records }}}\right)$ duplicate records.

Height $H_{i}$ of $R_{i}$ can be computed using a node's average fanout $f$ and the total number of records $T_{i} \cdot C_{i}$ as follows [2]:

$$
H_{i}=\left\lceil\log _{f}\left(T_{i} \cdot C_{i}\right)\right\rceil
$$




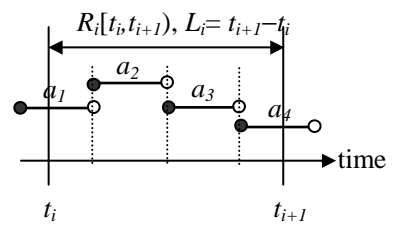

(a) $a_{1}$ and $a_{4}$ are fragmented

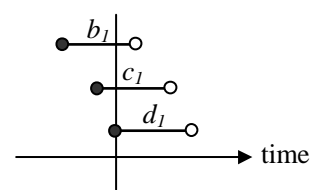

(b) $d_{1}$ is not fragmented

Fig. 11. Fragmentation of records by boundaries

From Formulas (1), (2), (3), and (4), the size of the APR-tree is:

$$
\text { Size }(A P R-\text { tree })=\sum_{i=1}^{K} \sum_{h=1}^{H_{i}} \frac{\left(\frac{L_{i}}{A V G_{\text {records }}}+\left(1-\frac{1}{A V G_{\text {records }}}\right)\right) \cdot C_{i}}{f^{h}}
$$

Let $\lambda_{i}$ be the ratio of duplicate records in $R_{i}$. Then, $\lambda_{i}$ can be defined as follows:

$$
\lambda_{i}=\frac{N_{\text {dup }}}{N_{\text {total }}}=\frac{\left(1-\frac{1}{A V G_{\text {records }}}\right) \cdot C_{i}}{\left(\frac{L_{i}}{A V G_{\text {records }}}+\left(1-\frac{1}{A V G_{\text {records }}}\right)\right) \cdot C_{i}} \leq \frac{1}{\frac{L_{i}}{A V G_{\text {records }}}+1}
$$

where $N_{d u p}$ is the number of duplicate records in $R_{i}$ and $N_{\text {total }}$ is the total number of records in $R_{i}$.

In Formula (6), $L_{i}$ determines $\lambda_{i}$ since $A V G_{\text {records }}$ is the average lifespan length of records and is obtained from records. Consequently, the larger $L_{i}$, the smaller $\lambda_{i}$. In terms of the size of the APR-tree, the larger $L_{i}$ results in the smaller size of the APR-tree. Extremely, when $L_{i}=\infty$, the APR-tree has no duplicate records. Nevertheless, we should allow duplicate records for reducing the search space of timestamp and interval queries.

\subsection{Estimation of the query cost of the APR-tree}

To estimate the interval query cost of the APR-tree, we extend the range query cost model of the R-tree $[2,15,18]$ since interval queries can be considered as range queries in the $\mathrm{R}$-tree. When an interval query overlaps the jurisdiction interval of $R_{i}$, we estimate the number of nodes accessed to answer this interval query in $R_{i}$. Assume that $L_{i}=\frac{1}{K}$ since the number of $R_{i}$ 's is $K$.

Let $M_{h}$ denote the number of nodes at height $h$ and $N_{i}$ denote the total number of records in $R_{i} . M_{h}$ can be computed by $N_{i}$ and as follows [2]:

$$
M_{h}=\left\lceil\frac{N_{i}}{f^{h}}\right\rceil \text { where } N_{i}=\left(\frac{L_{i}}{A V G_{\text {records }}}+\left(1-\frac{1}{A V G_{\text {records }}}\right)\right) \cdot C_{i}
$$


For example, for the leaf level, $M_{1}=\left\lceil\frac{N_{i}}{f}\right\rceil$.

Let $a_{h}$ denote the average area covered by a node at height $h$. Since $R_{i}$ covers $1 / \mathrm{K}$ of the d-dimensional work space $W S=[0,1)^{d}$ as shown in Figure 12, we have:

$$
a_{h}=\frac{1}{K} \cdot \frac{1}{M_{h}}
$$
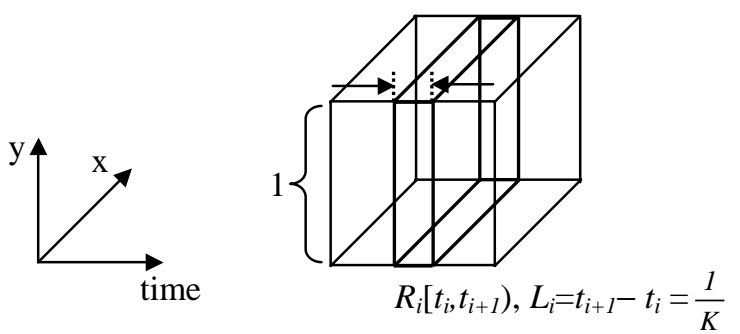

Fig. 12. $R_{i}$ in $W S=[0,1)^{d}$

Using the Minkowski sum technique $[2,14,17]$, the probability that a node at height $h$ may overlap the interval query rectangle $s$ is:

$$
\left(\sqrt[d]{s}+\sqrt[d]{a_{h}}\right)^{d}
$$

where $\sqrt[d]{s}$ and $\sqrt[d]{a_{h}}$ denote the average one-side lengths of $s$ and $a_{h}$, respectively. Therefore, the number of nodes at height $h$ that may overlap the query rectangle $s$ is:

$$
M_{h} \cdot\left(\sqrt[d]{s}+\sqrt[d]{a_{h}}\right)^{d}=\left(\sqrt[d]{\left\lceil\frac{N_{i}}{f^{h}}\right\rceil \cdot s}+\sqrt[d]{\frac{1}{K}}\right)^{d}
$$

where $M_{h}$ and $a_{h}$ are substituted with $N_{i}, f^{h}$, and $K$ by Formulas (7) and (8).

Let $N A_{i}$ be the total number of nodes accessed to answer the query rectangle $s$ in $R_{i} . N A_{i}$ is computed by summing Formula (10) from the leaf to the root as follows:

$$
N A_{i}=1+\sum_{h=1}^{\left\lceil\log _{f} N_{i}\right\rceil-1}\left(\sqrt[d]{\left\lceil\frac{N_{i}}{\left.f^{h}\right\rceil} \cdot s\right.}+\sqrt[d]{\frac{1}{K}}\right)^{d}
$$

When the interval query intersects the boundary, all the $R_{i}$ 's which overlap the interval query should be searched. Therefore, the query cost of the APR-tree is the sum of query costs of these $R_{i}$ 's which overlap the interval query. 
In this section, we present how to determine the tuning parameter $L_{i}$ which affects the query cost and the size. This procedure is based on the mathematical rationale using Formulas (6) and (11).
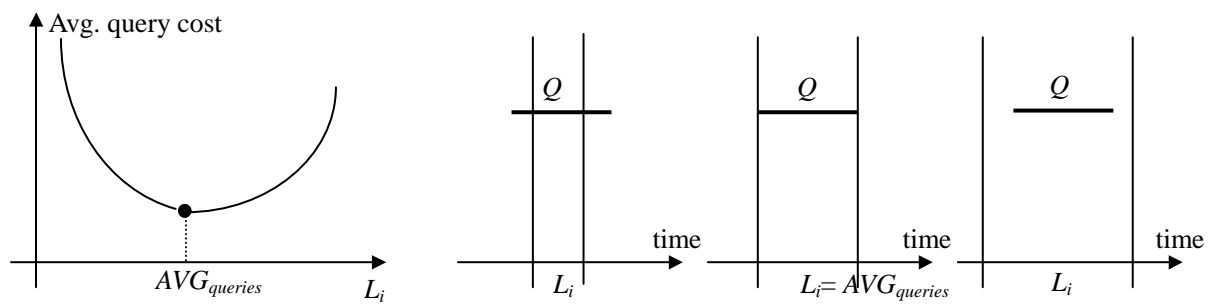

$\begin{array}{lll}\text { (i) } L_{i}<A V G_{\text {queries }} & \text { (ii) } L_{i}=A V G_{\text {queries }} & \text { (iii) } L_{i}>A V G_{\text {queries }}\end{array}$

Fig. 13. Average query cost for interval query $Q$ as a function of $L_{i}$

Figure 13 shows the average number of nodes accessed to answer an interval query $Q$ whose time interval length is $A V G_{\text {queries }}$ as a function of $L_{i}$. If the interval query $Q$ does not intersect the boundary, $L_{i}=A V G_{\text {queries }}$ makes the query cost smallest since the search space is smallest. When $L_{i}<A V G_{\text {queries }}$, the query cost increases since many $R_{i}$ 's should be searched. Conversely, when $L_{i}>A V G_{\text {queries }}$, the query cost also increases due to the increase of the search space. In other words, if $L_{i}<A V G_{\text {queries }}$, the query cost is affected by the number of $R_{i}$ 's which are searched. Since $L_{i}<A V G_{\text {queries }}$, the number of $R_{i}$ 's which are searched is at least 2 . The smaller $L_{i}$ decreases the search space, but increases the number of $R_{i}$ 's which are searched. In contrast, if $L_{i}>A V G_{\text {queries }}$, the query cost is affected by the search space. Since $L_{i}>$ $A V G_{\text {queries }}$, the number of $R_{i}$ 's which are searched is at most 2. Consequently, if $L_{i} \leq A V G_{\text {queries }}, L_{i}$ is chosen to minimize the number of $R_{i}$ 's which are searched. If $L_{i} \geq A V G_{\text {queries }}, L_{i}$ is chosen to minimize the search space.

Let $L_{i}=\alpha \cdot A V G_{\text {queries }}(\alpha>0)$. We can consider the following two cases according to the range of $\alpha$ : (i) $\alpha \leq 1$ (ii) $\alpha \geq 1$. Namely, $\alpha \leq 1$ means that $L_{i} \leq A V G_{\text {queries }}$, and $\alpha \geq 1$ means that $L_{i} \geq A V G_{\text {queries }}$.

If $\alpha \leq 1$ (i.e., $L_{i} \leq A V G_{\text {queries }}$ ), the query cost depends on the number of $R_{i}$ 's which are searched. Since the smaller $L_{i}$ increases the number of $R_{i}$ 's which are searched, $\alpha=1$ (i.e., $L_{i}=A V G_{\text {queries }}$ ) minimizes the number of $R_{i}$ 's which are searched. When $L_{i}=A V G_{\text {queries }}$, the number of $R_{i}$ 's which are searched is at most 2. Consequently, if $L_{i} \leq A V G_{\text {queries }}, L_{i}=A V G_{\text {queries }}$ minimizes the query cost.

If $\alpha \geq 1$ (i.e., $L_{i} \geq A V G_{\text {queries }}$ ), the query cost depends on the search space. As shown in Figure 14, there are the following two cases: The first case is that the interval query $Q$ does not intersect the boundary. The second case is 


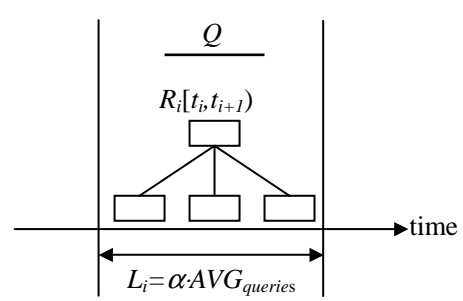

(a) $S_{\text {not_intersect }}=\alpha \cdot A V G_{\text {queries }}$

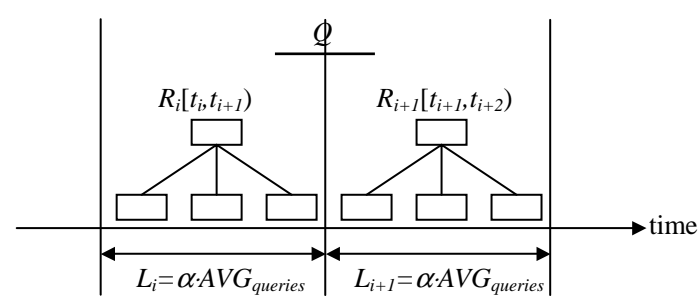

(b) $S_{\text {intersect }}=2 \alpha \cdot A V G_{\text {queries }}$

Fig. 14. Difference between search spaces of two cases

that the interval query $Q$ intersects the boundary. Therefore, $L_{i}$ is chosen to minimize the average search space of two cases.

Let $P_{\text {intersect }}$ be the probability that the interval query $Q$ intersects the boundary. Since $P_{\text {intersect }}$ is the same as the probability that an interval whose length is $A V G_{\text {queries }}$ intersects a point within the range of $\left[0, L_{i}\right)$, we have:

$$
P_{\text {intersect }}=\frac{A V G_{\text {queries }}}{L_{i}}
$$

For example, if $L_{i}=2 \cdot A V G_{\text {queries }}, P_{\text {intersect }}=0.5$.

Let $P_{\text {not_intersect }}$ be the probability that the interval query $Q$ does not intersect the boundary. Since $P_{\text {not_intersect }}=1-P_{\text {intersect }}$, we have:

$$
P_{\text {not_intersect }}=1-\frac{A V G_{\text {queries }}}{L_{i}}
$$

Let $S_{\text {not_intersect }}$ denote the search space of the APR-tree when the interval query $Q$ does not intersect the boundary. As shown in Figure 14(a), since the interval query $Q$ intersects only $R_{i}$ and $L_{i}=\alpha \cdot A V G_{\text {queries }}$, the search space of the APR-tree is:

$$
S_{\text {not_intersect }}=\alpha \cdot A V G_{\text {queries }}
$$

Let $S_{\text {intersect }}$ denote the search space of the APR-tree when the interval query $Q$ intersects the boundary. As shown in Figure 14(b), since the interval query $Q$ intersects both $R_{i}$ and $R_{i+1}$, and $L_{i}=L_{i+1}=\alpha \cdot A V G_{\text {queries }}$, the search space of the APR-tree is:

$$
S_{\text {intersect }}=2 \alpha \cdot A V G_{\text {queries }}
$$

Let $S_{\text {avg }}$ denote the average search space of the APR-tree for the two cases as shown in Figure 14. From Formulas (12), (13), (14), and (15), the average search space of the APR-tree is:

$$
S_{\text {avg }}=P_{\text {intersect }} \cdot S_{\text {intersect }}+P_{\text {not_intersect }} \cdot S_{\text {not_intersect }}=(\alpha+1) \cdot A V G_{\text {queries }}
$$


In Formula (16), since $\alpha \geq 1, \alpha=1$ minimizes the average search space of the APR-tree. When $L_{i}=A V G_{\text {queries }}, S_{\text {avg }}=2 \cdot A V G_{\text {queries }}$. Consequently, if $L_{i} \geq A V G_{\text {queries }}, L_{i}=A V G_{\text {queries }}$ minimizes the query cost. As a result, $L_{i}=A V G_{\text {queries }}$ reduces the number of $R_{i}$ 's which are searched to 2 . and the average search space of the APR-tree is minimized. Therefore, $L_{i}=$ $A V G_{\text {queries }}$ minimizes the average cost of the query $Q$ whose time interval length is $A V G_{\text {queries }}$.

As shown in Formula (6), $L_{i}$ determines the ratio of duplicate records. In other words, the smaller $L_{i}$, the larger size of the APR-tree. Figure 15 illustrates the size of the APR-tree as a function of $L_{i}$. In this figure, the difference between Size (3DR-tree) and Size $(A P R-$ tree $)$ is due to duplicate records spawned by the boundary. To prevent that the size of the APR-tree becomes prohibitively large, $L_{i}$ should have the minimum threshold. Therefore, we use $A V G_{\text {records }}$ as the minimum value of $L_{i} . L_{i} \geq A V G_{\text {records }}$ assures that the size of the APR-tree does not exceed 2 times that of the 3DR-tree since by Formula (6), when $L_{i}=A V G_{\text {records }}, \lambda_{i} \leq 0.5$. Namely, $\lambda_{i}=0.5$ means that most of records intersect boundaries, so the number of duplicate records nearly equals the number of non-duplicate records.

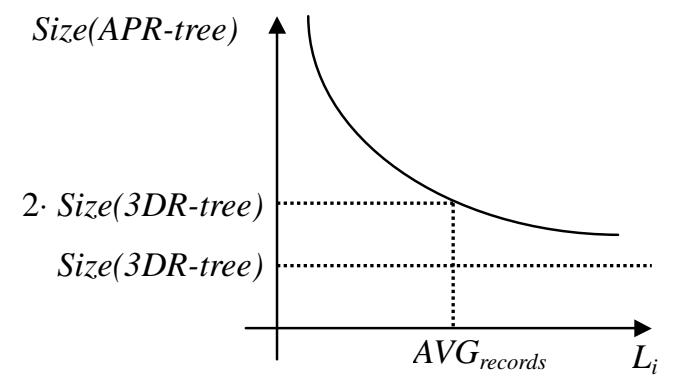

Fig. 15. The size of the APR-tree as a function of $L_{i}$

To determine $L_{i}$ dynamically, we take advantage of query workloads. Figure 16 shows the algorithms to compute $A V G_{\text {queries }}$ and $A V G_{\text {records }}$. They are recomputed when queries and records are entered so that they can be dynamically adjusted. In Figure 16, $N_{\text {queries }}$ and $N_{\text {records }}$ denote the numbers of queries and records, respectively.

Using $A V G_{\text {queries }}$ and $A V G_{\text {records }}$ obtained from the query workload and the dataset, respectively, the tuning parameter $L_{i}$ is dynamically determined by the following formula.

$$
L_{i}=\max \left(A V G_{\text {queries }}, A V G_{\text {records }}\right)
$$

where $\max \left(A V G_{\text {queries }}, A V G_{\text {records }}\right)$ chooses the larger value between $A V G_{\text {queries }}$ and $A V G_{\text {records }}$.

Formula (17) guarantees the following two things: The first is that the size of the APR-tree does not exceed 2 times that of the 3DR-tree since $L_{i} \geq$ 
proc Compute_AVG $G_{\text {queries }}\left(\right.$ query $q=<S,\left[t_{0}, t_{1}\right)$, results $>$ )

begin

1. $A V G_{\text {queries }}:=\frac{N_{\text {queries }} \times A V G_{\text {queries }}+\left(t_{1}-t_{0}\right)}{N_{\text {queries }}+1}$

2. $N_{\text {queries }}:=N_{\text {queries }}+1$

end

proc Compute_AVG $G_{\text {records }}\left(\right.$ record $r=<i d, s,\left[t_{0}, t_{1}\right)>$ )

begin

1. $A V G_{\text {records }}:=\frac{N_{\text {records }} \times A V G_{\text {records }}+\left(t_{1}-t_{0}\right)}{N_{\text {records }}+1}$

2. $N_{\text {records }}:=N_{\text {records }}+1$

end

Fig. 16. Computation of $A V G_{\text {queries }}$ and $A V G_{\text {records }}$

$A V G_{\text {records. }}$. The second is that when $A V G_{\text {queries }} \geq A V G_{\text {records }}$, the query cost is smallest since $L_{i}=A V G_{\text {queries }}$. However, when $A V G_{\text {queries }}<A V G_{\text {records }}$, the query cost is not smallest since $L_{i}>A V G_{\text {queries }}$ and the APR-tree suffers from the enlarged search space. However, the performance of the APR-tree would not degrade unless $A V G_{\text {records }}$ is much larger than $A V G_{\text {queries }}$.

\section{Experiments}

In this section, we compare the APR-tree with the HR-tree, the 3DR-tree and the MV3R-tree in terms of query cost, size, and update cost. Section 5.1 describes the experimental environment and Section 5.2 provides the experimental results.

\subsection{Experimental Environment}

Due to the lack of real data, synthetic datasets with real world semantics are generated by the GSTD method [13] which has been widely employed (e.g., $[8,9,11,12])$ as a benchmarking environment for access methods handling moving points and regions. Objects change their shapes or positions randomly. We investigate their movements for 500 timestamps. The cardinality of objects is 10,000 . Objects are uniformly distributed in the spatial area. Agilities of datasets are $10 \%, 20 \%$, and $30 \%$, where the agility denotes the ratio of objects which change their positions or shapes at each timestamp. For example, agility $=10 \%$ means that $10 \%$ of the objects change their positions or shapes at each timestamp. To estimate $A V G_{\text {queries }}, 10 \%$ of queries in the workload are randomly selected. $A V G_{\text {records }}$ is computed using records at the index building time. 
Note that in this work, the R-tree implementations of all the spatio-temporal access methods are based on algorithms of the $\mathrm{R}^{*}$-tree [16]. The values of parameters of the MV3R-tree are the same as those in [11]: $P_{w v}=0.35$ and $P_{s v o}=0.85$, where $P_{w v}$ indicates the value for the weak version condition and $P_{s v o}$ indicates the value for the strong version overflow condition. A node corresponds to a page, whose size is 1 KBytes. Using this size, the maximum fanouts of the HR-tree, the 3DR-tree, the MV3R-tree, and the APR-tree are $50,36,36$, and 36 , respectively. In the HR-tree, the other nodes except root nodes do not keep temporal information, so the maximum fanout of the HRtree is larger than those of the other access methods.

To simulate real life situations, the five query workloads in Table 3 are employed. Each workload has 1,000 queries. Spatial areas of queries are 1\%, 3\%, $5 \%, 7 \%$, and $9 \%$ of the whole spatial area. The maximum time interval length of queries are between $1 \%(5=500 \times 1 \%)$ and $20 \%(100=500 \times 20 \%)$ of whole time interval.

Table 3

Five workloads which consist of timestamp and interval queries

\begin{tabular}{|c|c|c|}
\hline & Timestamp queries & Interval queries \\
\hline \hline The $1^{\text {st }}$ workload & $100 \%$ & $0 \%$ \\
\hline The $2^{\text {nd }}$ workload & $75 \%$ & $25 \%$ \\
\hline The $3^{r d}$ workload & $50 \%$ & $50 \%$ \\
\hline The $4^{t h}$ workload & $25 \%$ & $75 \%$ \\
\hline The $5^{\text {th }}$ workload & $0 \%$ & $100 \%$ \\
\hline
\end{tabular}

To show the efficiency of the APR-tree, we measure the average number of nodes accessed to answer timestamp and interval queries in the workload. The justification for performance metric is that the smaller number of node accesses, the fewer disk I/O's needed to evaluate timestamp and interval queries.

\subsection{Experimental Results}

Figure 17 shows the performance of each access method for various query workloads when the dataset agility is $20 \%$. Since the experimental results for dataset agilities $=10 \%$ and $30 \%$ are similar to those for the dataset agility $=$ $20 \%$, the experimental results for dataset agilities $=10 \%$ and $30 \%$ are omitted. For the workload with $0 \%$ interval queries (i.e., $100 \%$ timestamp queries), the 3DR-tree shows the worst performance, and the HR-tree shows the best performance since the search space of the HR-tree is optimal for timestamp queries and the average fanout of the HR-tree is much larger than those of the other access methods. Conversely, for the workload with $100 \%$ interval queries, the HR-tree shows the worst performance since the HR-tree should search many R-trees. The MV3R-tree outperforms the HR-tree and the 3DR-tree for 

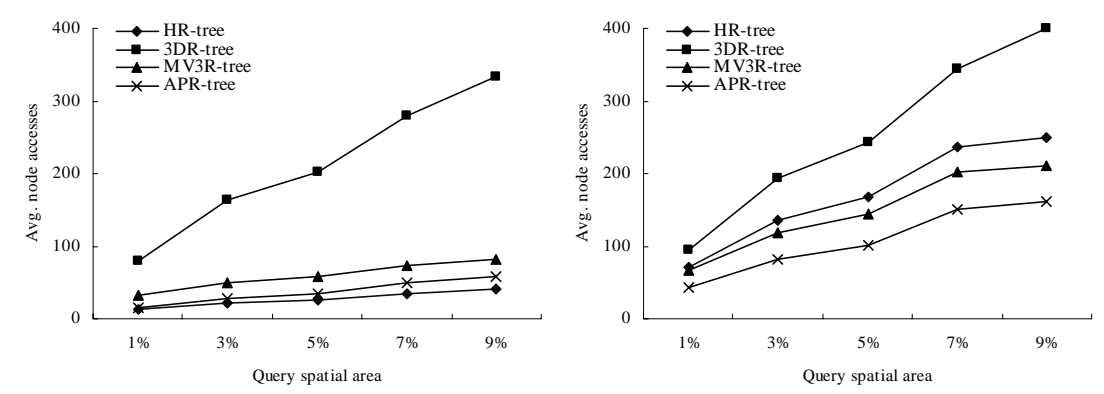

(a) $0 \%$ interval queries

(b) $25 \%$ interval queries
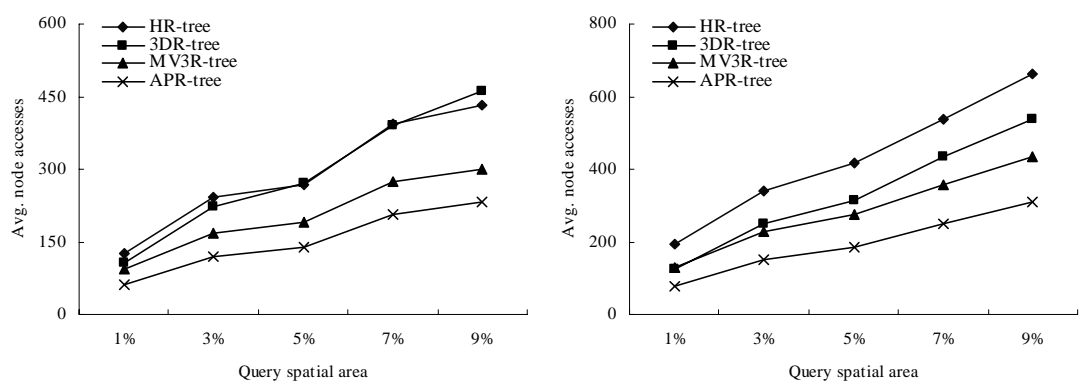

(c) $50 \%$ interval queries

(d) $75 \%$ interval queries

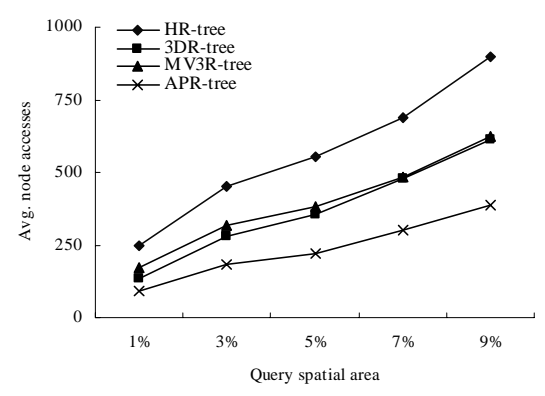

(e) $100 \%$ interval queries

Fig. 17. Query costs for various query workloads (agility $=20 \%$ )

the workloads with $25 \%, 50 \%$, and $75 \%$ interval queries since the MV3R-tree combines the MVR-tree for timestamp queries and the auxiliary 3DR-tree for interval queries. Thus, for the workload with $100 \%$ interval queries, the performance of the MV3R-tree is similar to that of the 3DR-tree since the auxiliary 3DR-tree also searches the whole history like the 3DR-tree. For the workloads with above $25 \%$ interval queries, the APR-tree achieves the best performance since $A V G_{\text {queries }}>A V G_{\text {records }}$ and $L_{i}=A V G_{\text {queries }}$. However, for the workload with $0 \%$ interval queries, the performance of the APR-tree is worse than that of the HR-tree since the search space of the APR-tree is larger than that of the HR-tree and the average fanout of the APR-tree is smaller than that of the HR-tree. However, as shown in Figures 17(b), 17(c), $17(\mathrm{~d})$, and 17(e), the APR-tree outperforms the other access methods for a variety of query workloads.

Figure 18 shows the performance of each access method for the workload with 


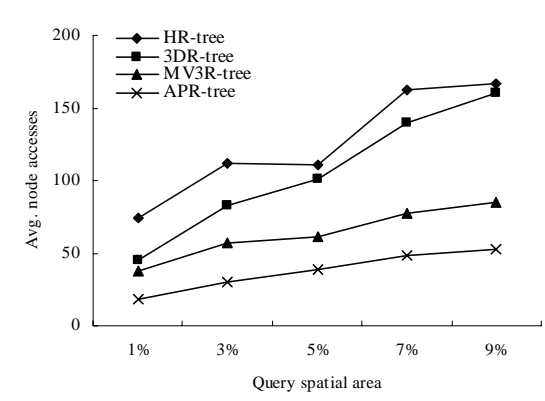

(a) Agility $=1 \%$

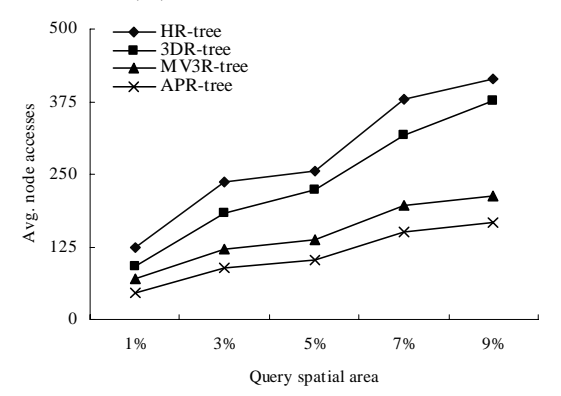

(c) Agility $=10 \%$

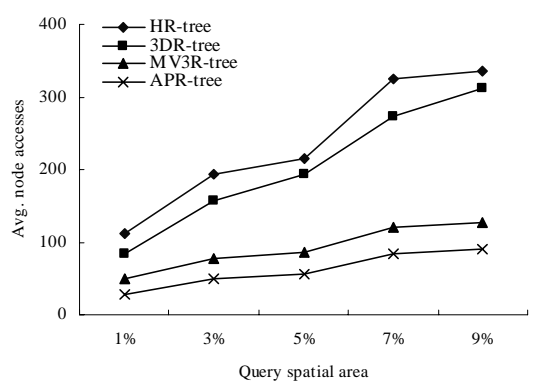

(b) Agility $=5 \%$

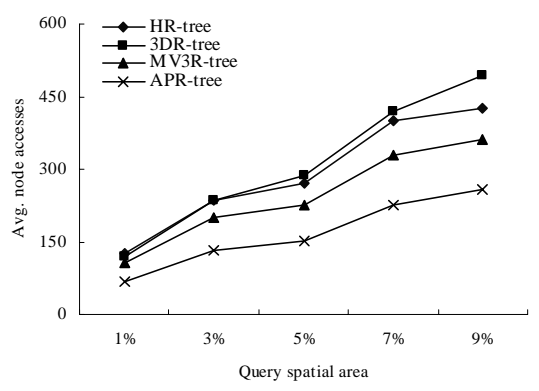

(d) Agility $=30 \%$

Fig. 18. Query costs for various agilities (50\% interval queries)

$50 \%$ interval queries when the dataset agility varies from $1 \%$ to $30 \%$. Note that Figure 17(c) shows the result for the workload with $50 \%$ interval queries when dataset agility is $10 \%$. Regardless of the dataset agility, the APR-tree accomplishes the best performance for this workload since $A V G_{\text {queries }}>A V G_{\text {records }}$ and $L_{i}=A V G_{\text {queries }}$. When the dataset agility is $30 \%$, the APR-tree outperforms the other access methods. This is due to the fact that $A V G_{\text {records }}$ decreases as the agility increases. By Formula (6), the smaller $A V G_{\text {records }}$ results in the smaller number of duplicate records. By Formula (11), the smaller number of duplicate records improves the performance of the APR-tree since the total number of records decreases. Regardless of the dataset agility, the MV3R-tree also outperforms the HR-tree and the 3DR-tree since the MV3Rtree combines the MVR-tree responsible for timestamp queries and the auxiliary 3DR-tree responsible for interval queries. Since the number of duplicate data decreases as the agility increases, this improves the performance of the HR-tree. When the dataset agility is $10 \%$, the 3DR-tree outperforms the HRtree. In contrast, when the dataset agility is 30\%, the HR-tree shows as similar performance as the 3DR-tree.

Figure 19 shows the performance of each access method for the workload with $100 \%$ interval queries when the dataset agility is $20 \%$ and maximum interval length of queries varies from $5(=500 \times 1 \%)$ to $100(=500 \times 20 \%)$. Note that Figure 17(e) shows the result for the workload with $100 \%$ interval queries when the dataset agility is $20 \%$ and maximum interval length of queries is 50 $(=500 \times 10 \%)$. When maximum interval length is 5 as shown in Figure 19(a), the 3DR-tree shows the worst performance due to a large search space while 


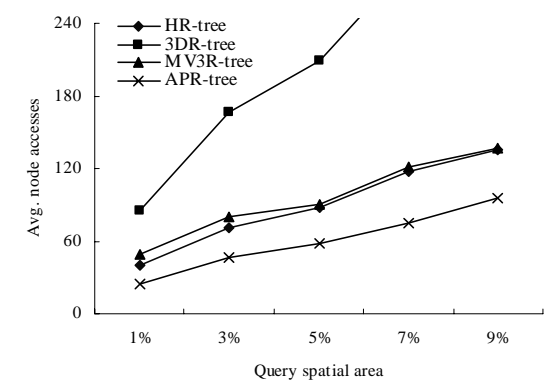

(a) Maximum length $=5(1 \%)$

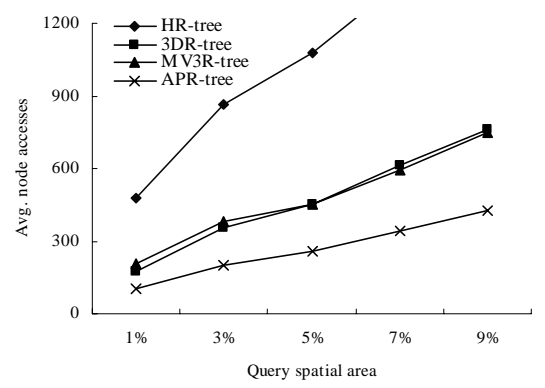

(b) Maximum length $=100(20 \%)$

Fig. 19. Query costs for various maximum interval lengths (agility $=20 \%$ and $100 \%$ interval queries)

the HR-tree and the MV3R-tree yield similar performance. In contrast, when maximum interval length is 100 as shown in Figure 19(b), the HR-tree shows the worst performance due to an access to a large number of R-trees while the 3DR-tree and the MV3R-tree yield similar performance. The APR-tree outperforms the others for various maximum interval length since it employs the query adaptive index scheme.

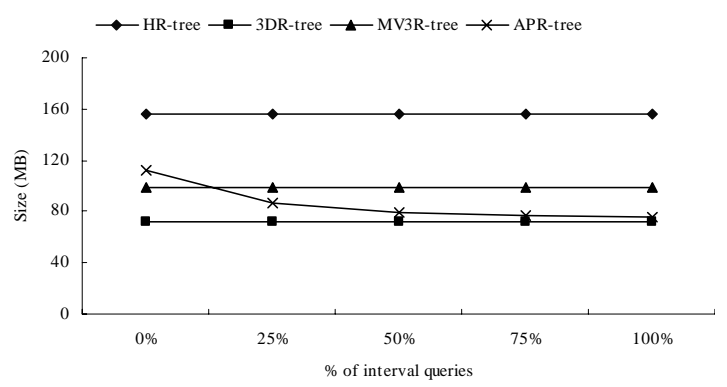

Fig. 20. The size comparison for various query workloads (agility $=20 \%$ )

Figure 20 shows the size of each access method for various query workloads when the dataset agility is $20 \%$. The 3DR-tree has the smallest size since it has no duplicate data. The size of the HR-tree is about 2.7 times larger than that of the 3DR-tree since it has a huge number of duplicate data. The size of the MV3R-tree is about 1.5 times larger than that of 3DR-tree since it has a large number of duplicate data due to version splits. As shown in Figure 20, the sizes of the HR-tree, the 3DR-tree and the MV3R-tree are determined by datasets. In other words, the sizes of these access methods are not affected by query workloads. In contrast, the size of the APR-tree is associated with query workloads as well as datasets since $L_{i}$ is determined by $A V G_{\text {queries }}$ and $A V G_{\text {records. }}$. The size of the APR-tree is on the average 1.3 times larger than that of the 3DR-tree. As the ratio of timestamp queries increases, the size of the APR-tree increases due to the smaller $L_{i}$. For the workload with $0 \%$ interval queries (i.e., $100 \%$ timestamp queries), the size of the APR-tree is about 1.7 times larger than that of the $3 \mathrm{DR}$-tree since $A V G_{\text {queries }}=1$ is smaller than $A V G_{\text {records }}$ and by Formula (17), $L_{i}=A V G_{\text {records }}$ causes a large number of duplicate records. Although for the workload with $0 \%$ interval queries, 
the size of the APR-tree is larger than that of the MV3R-tree, the APR-tree outperforms the MV3R-tree as shown in Figure 17(a). For the workloads with above $25 \%$ interval queries, the size of the APR-tree is smaller than that of the MV3R-tree. Especially, for the workload with 100\% interval queries, the size of the APR-tree approaches to that of the 3DR-tree.

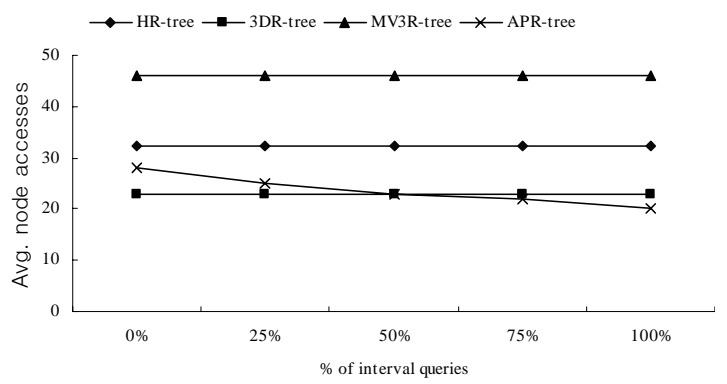

Fig. 21. Update costs for various query workloads (agility $=20 \%$ )

Figure 21 shows the update cost of each access method for various query workloads when the dataset agility is $20 \%$. The update cost denotes the average number of nodes accessed to update each structure for object changes (insertion and logical deletion) and is measured by using the following equation: Update cost $=\frac{N_{2}}{N_{1}}$ where $N_{1}$ denotes the total number of records and $N_{2}$ denotes the total number of nodes accessed to insert $N_{1}$ records.

Compared with the HR-tree and the MV3R-tree, the update cost of the 3DRtree is smallest since it has the smallest size and maintains the single structure. The update cost of the MV3R-tree is typically 2 times higher than that of the 3DR-tree since the MV3R-tree has to modify the MVR-tree and the auxiliary 3DR-tree concurrently. The update cost of the HR-tree is about 1.4 times higher than that of the 3DR-tree since the size of the HR-tree is large and nodes in the path between the leaf and the root node may be modified or created even when an object changes its shape or position. Since the size and the height of the APR-tree change according to query workloads, the update cost of the APR-tree is also affected by query workloads. Since the height of the APR-tree is lower than that of the 3DR-tree and the size of the APR-tree is on the average 1.3 times larger than that of the 3DR-tree, the update cost of the APR-tree is on the average similar to that of the 3DR-tree. As the ratio of interval queries increases, the size and the update cost of the APR-tree decrease since $L_{i}$ increases.

\section{Conclusions}

To overcome the problems with the HR-tree, the 3DR-tree and the MV3Rtree for timestamp and interval queries, we proposed the APR-tree which takes 
account of query workloads. Since the jurisdiction interval length of the APRtree is automatically fitted to the query workload, the APR-tree outperforms the other access methods for various query workloads. Unlike the other access methods, the size and the update cost of the APR-tree are affected by query workloads. As the ratio of interval queries increases, the size and the update cost of the APR-tree decrease. The size of the APR-tree is on the average 1.3 times larger than that of the 3DR-tree. The update cost of the APR-tree is on the average similar to that of the 3DR-tree. Additionally, since the HRtree and the MV3R-tree have the graph structure, when dealing with interval queries, they require additional overheads, such as the maintenance of the access list in order to avoid re-accessing nodes. However, since the 3DR-tree and the APR-tree have the tree structure, they do not require such overheads.

\section{References}

[1] G. Kollios, D. Gunopulos, and V. J. Tsotras, A. Delis, and M. Hadjielefteriou: Indexing Animated Objects Using Spatiotemporal Access Methods, TKDE $13(5), 2001$.

[2] Y. Theodoridis and T. K. Sellis: A Model for the Prediction of R-tree Performance. ACM PODS, 1996.

[3] M. A. Nascimento and J. R. O. Silva: Towards historical R-trees. ACM SAC, 1998.

[4] B. Becker, S. Gschwind, T. Ohler, B. Seeger, and P. Widmayer: An Asymptotically Optimal Multiversion B-Tree. VLDB Journal 5(4), 1996.

[5] P. K. Agarwal, L. Arge, and J. Erickson: Indexing Moving Points. ACM PODS, 2000 .

[6] G. Kollios, D. Gunopulos, and V. J. Tsotras: On Indexing Mobile Objects. ACM PODS, 1999.

[7] S. Saltenis, C. S. Jensen, S. T. Leutenegger, and M. A. Lopez: Indexing the Positions of Continuously Moving Objects. ACM SIGMOD, 2000.

[8] D. Pfoser, C. S. Jensen, and Y. Theodoridis: Novel Approaches in Query Processing for Moving Object Trajectories. VLDB, 2000.

[9] Y. Tao and D. Papadias: Efficient Historical R-Trees. SSDBM, 2001.

[10] M. Vazirgiannis, Y. Theodoridis, and T. K. Sellis: Spatio-Temporal Composition and Indexing for Large Multimedia Applications. Multimedia Systems 6(4), 1998.

[11] Y. Tao and D. Papadias: MV3R-Tree: A Spatio-Temporal Access Method for Timestamp and Interval Queries. VLDB, 2001. 
[12] M. A. Nascimento, J. R. O. Silva, and Y. Theodoridis: Evaluation of Access Structures for Discretely Moving Points. Spatio-Temporal Database Management, 1999.

[13] Y. Theodoridis, J. R. O. Silva, and M. A. Nascimento: On the Generation of Spatiotemporal Datasets. SSD, 1999.

[14] I. Kamel and C. Faloutsos: On Packing R-trees. CIKM, 1993.

[15] A. Guttman: R-Trees: A Dynamic Index Structure for Spatial Searching. ACM SIGMOD, 1984.

[16] N. Beckmann, H. Kriegel, R. Schneider, and B. Seeger: The R*-Tree: An Efficient and Robust Access Method for Points and Rectangles. ACM SIGMOD, 1990.

[17] Y. Theodoridis, E. Stefanakis, and T. K. Sellis: Efficient Cost Models for Spatial Queries Using R-Trees. TKDE 12(1), 2000. 